\title{
A vital sign-based prediction algorithm for differentiating COVID-19 versus seasonal influenza in hospitalized patients
}

\author{
Naveena Yanamala (iD ${ }^{1,2 凶}$, Nanda H. Krishna (iD ${ }^{1,2}$, Quincy A. Hathaway ID) $^{1}$ Aditya Radhakrishnan ${ }^{1,2}$, Srinidhi Sunkara ${ }^{1,2}$, \\ Heenaben Patel ${ }^{1}$, Peter Farjo ${ }^{1}$, Brijesh Patel ${ }^{1}$ and Partho P. Sengupta ${ }^{1 凶}$
}

Patients with influenza and SARS-CoV2/Coronavirus disease 2019 (COVID-19) infections have a different clinical course and outcomes. We developed and validated a supervised machine learning pipeline to distinguish the two viral infections using the available vital signs and demographic dataset from the first hospital/emergency room encounters of 3883 patients who had confirmed diagnoses of influenza A/B, COVID-19 or negative laboratory test results. The models were able to achieve an area under the receiver operating characteristic curve (ROC AUC) of at least $97 \%$ using our multiclass classifier. The predictive models were externally validated on 15,697 encounters in 3125 patients available on TrinetX database that contains patient-level data from different healthcare organizations. The influenza vs COVID-19-positive model had an AUC of 98.8\%, and 92.8\% on the internal and external test sets, respectively. Our study illustrates the potentials of machine-learning models for accurately distinguishing the two viral infections. The code is made available at https://github.com/ynaveena/COVID-19-vs-Influenza and may have utility as a frontline diagnostic tool to aid healthcare workers in triaging patients once the two viral infections start cocirculating in the communities.

npj Digital Medicine (2021)4:95 ; https://doi.org/10.1038/s41746-021-00467-8

\section{INTRODUCTION}

Infection with severe acute respiratory syndrome coronavirus 2 (SARS-CoV 2) causing coronavirus disease 2019 (COVID-19) has led to an unprecedented global crisis due to its vigorous transmission, spectrum of respiratory manifestations, and vascular affects ${ }^{1-3}$. The etiology of the disease is further complicated by a diverse set of clinical presentations, ranging from asymptomatic to progressive viral pneumonia and mortality ${ }^{4}$. Due to its similar symptomatology, COVID-19 has drawn comparisons to the seasonal influenza epidemic ${ }^{5}$. Both infections commonly present with overlapping symptoms, leading to a clinical dilemma for clinicians as SARS-CoV 2 carries a case-fatality rate up to 30 times that of influenza and infects healthcare workers at a significantly higher rate ${ }^{3,6,7}$. Moreover, the concurrence of epidemics appears imminent as the considerable COVID-19 incidence continues and even a moderate influenza season would result in over 35 million cases and 30,000 deaths ${ }^{5,6}$. To help curb this dilemma, front-line providers need the ability to rapidly and accurately triage these patients.

One approach to quickly classifying patients as COVID-19 positive or negative could be through machine learning algorithms. While the use of machine learning has been applied to contact tracing and forecasting during the COVID-19 epidemic ${ }^{8}$, it has only limitedly been explored as a means for accurately predicting COVID-19 infection on clinical presentation. With just a few important parameters clinicians can diagnose the patients well before a laboratory diagnosis. Preliminary work has shown the utility of machine and deep learning algorithms in predicting COVID-19 for patient features ${ }^{9-11}$ and on CT examination ${ }^{12,13}$, but there remains a paucity in research showing the capacity of machine learning algorithms in differentiating between COVID-19 and influenza patients.
Vital signs are critical piece of information used in the initial triage of patients with COVID-19 and/or influenza by care coordinators and health-care responders in community urgent care centers or emergency rooms. It is becoming clearer that patient vital signs may present uniquely in SARS-CoV 2 infection ${ }^{9}$, likely as a result of alterations in gas exchange and microvascular changes ${ }^{14}$. In the present investigation, we therefore explored the use of machine learning models to differentiate between SARS-CoV 2 and influenza infection using basic officebased clinical variables. The use of simple ML-based classification may have utility for the rapid identification, triage, and treatment of COVID-19 and influenza-positive patients by front-line healthcare workers, which is especially relevant as the influenza season approaches.

\section{RESULTS \\ WVU study cohort}

The WVU study patient cohort included 3883 patients (mean age $52 \pm 24$ years, $48 \%$ males, and $89 \%$ White/Caucasian) of whom 747 (19\%) tested positive for SARSCoV-2 (COVID-19 positive cohort), 1913 (49\%) tested negative for SARSCoV-2 (COVID-19 negative cohort), and 1223 (31\%) had influenza (Table 1, Supplementary Fig. 1). The majority of the COVID-19 positive and negative patients were older, whereas the influenza cohort was younger $(P<0.001)$. There was higher prevalence of Black/African Americans in the influenza cohort in comparison with other cohorts. COVID-19 positive patients were more obese $(p<0.001)$. They also had higher mean body temperature compared to COVID-negative patients and exhibited an overall higher systolic and diastolic blood pressures than the other two cohorts ( $p<0.001$ for all variables). However, the influenza cohort had higher mean body temperature, heart rate, and oxygen saturations than COVID-19-positive and -negative

'Division of Cardiology, West Virginia University Medicine Heart \& Vascular Institute, Morgantown, WV, USA. ${ }^{2}$ Institute for Software Research, School of Computer Science, Carnegie Mellon University, Pittsburgh, PA, USA. ${ }^{凶}$ email: naveena.yanamala.m@wvumedicine.org; Partho.Sengupta@wvumedicine.org 
Table 1. Demographics of WVU patients (included COVID-19-positive, COVID-19-negative, and influenza cohort).

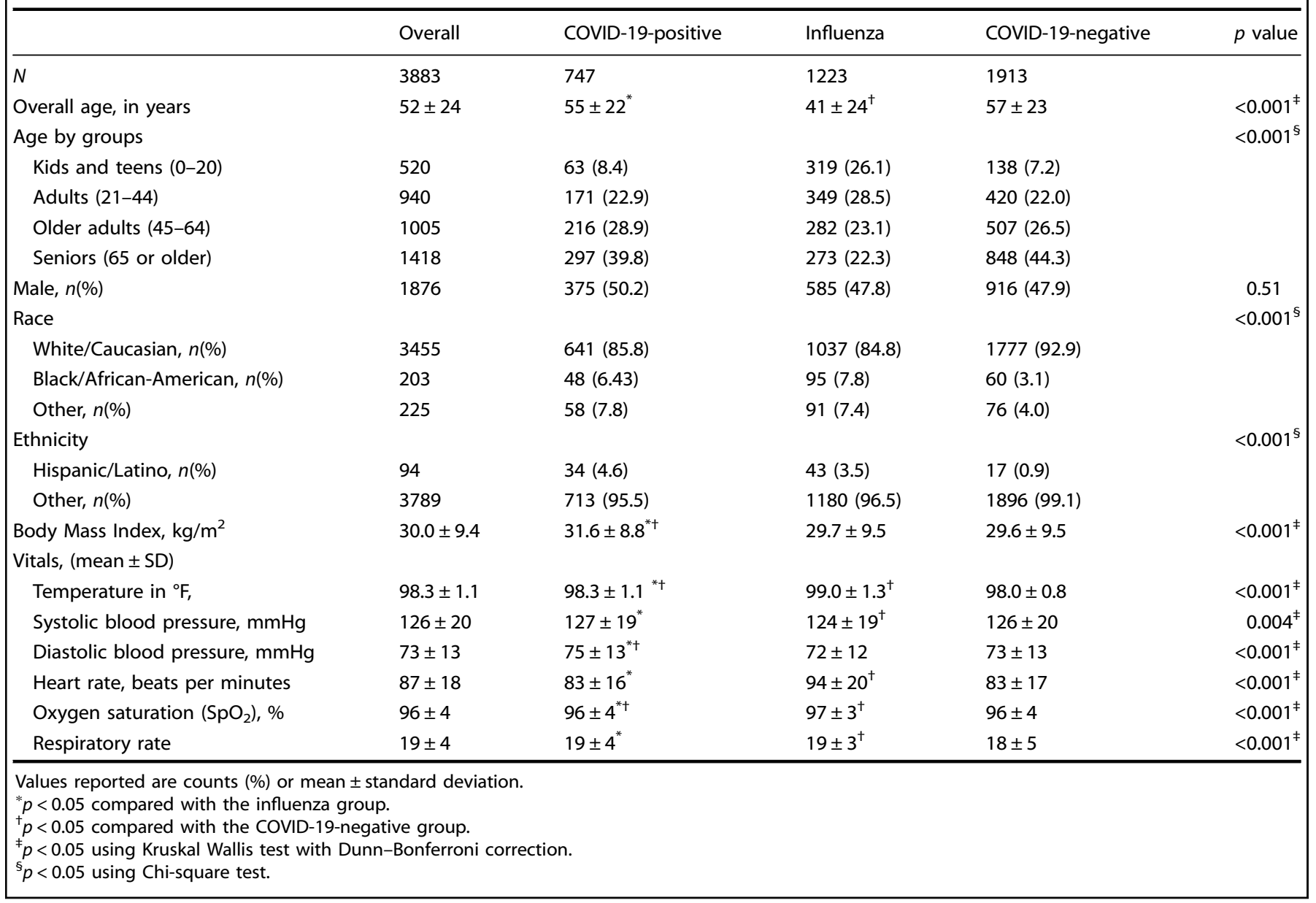

Table 2. Outcomes of WVU patients presented at ED or admitted to WVU hospitals and tested positive for COVID-19, positive for influenza A/B or negative for COVID-19.

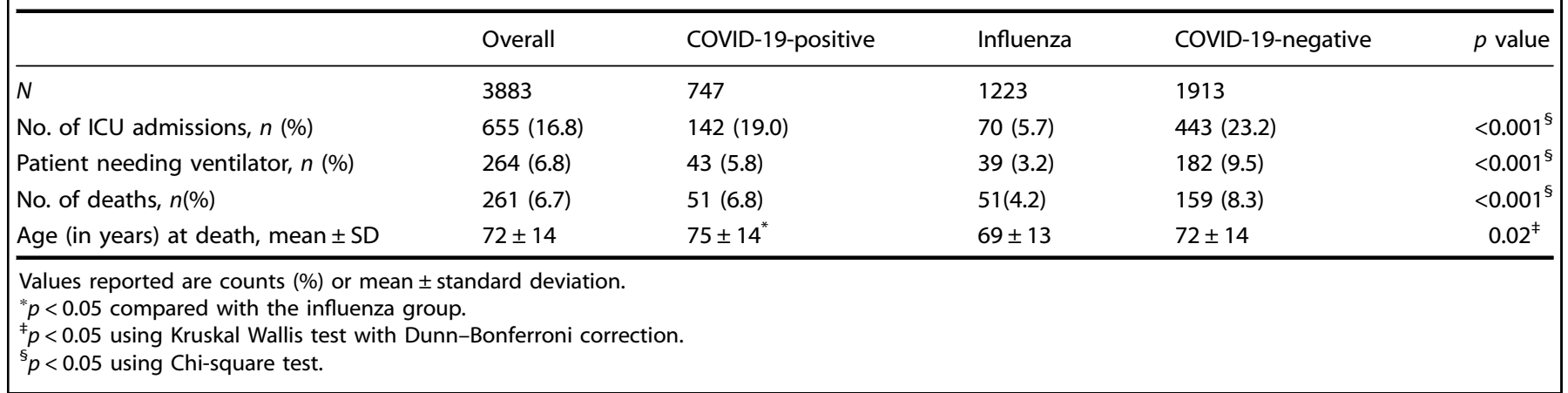

patients $(p<0.001)$. Patients in the COVID-19-positive and influenza groups had a higher respiratory rate than the COVID-19-negative group (Table 1).

The overall mortality for the WVU study cohort was $6.7 \%$. The crude case fatality rate was $6.8 \%$ in the COVID-19-positive and $4.2 \%$ in influenza groups, with a $9.5 \%$ case fatality rate in the COVID-19 negative group $(p<0.001)$. The COVID-19-positive patients had more than a 3-fold higher rate for ICU admissions than patients with influenza $(19.0 \%$ vs $5.7 \% ; p<0.001)$, but the rate was lower than that in the COVID-19 negative group (23.2\%) $(p<0.001)$. The average age of patients who died during hospitalizations was significantly higher in COVID-19 positive patients (75 \pm 14 years) compared to the influenza (69 \pm 13 years) and COVID-19 negative groups (72 \pm 14 years) $(p=0.02)$, as presented in Table 2.

\section{TriNetX cohort}

The TriNetX external validation cohort included a total of 15,697 patient encounters from 3125 patients with body temperature information available (Supplementary Table 2). This subgroup of the external cohort included 6613 encounters from 1057 COVIDpositive patients and 9087 encounters from 2068 influenza patients. The COVID-19-positive group was predominantly male 
Table 3. Performance metrics of different XGBoost models for predicting the given record as COVID-19-positive or influenza or COVID-19-negative when tested on internal validation or test set.

\begin{tabular}{llll}
\hline $\begin{array}{l}\text { Performance } \\
\text { measure }\end{array}$ & $\begin{array}{l}\text { COVID-19- } \\
\text { positive vs } \\
\text { influenza }\end{array}$ & $\begin{array}{l}\text { Influenza } \\
\text { vs others }\end{array}$ & $\begin{array}{l}\text { Covid-19- } \\
\text { positive vs } \\
\text { COVID-19- } \\
\text { negative }\end{array}$ \\
\hline AUC & 0.988 & 0.986 & 0.973 \\
Accuracy & 0.949 & 0.945 & 0.930 \\
F1 score & 0.928 & 0.918 & 0.878 \\
Sensitivity (recall) & 0.925 & 0.913 & 0.872 \\
Specificity & 0.962 & 0.961 & 0.954 \\
PPV (precision) & 0.932 & 0.924 & 0.884 \\
NPV & 0.958 & 0.955 & 0.945 \\
FPR & 0.038 & 0.039 & 0.046 \\
FDR & 0.069 & 0.076 & 0.116 \\
FNR & 0.075 & 0.087 & 0.128 \\
\hline
\end{tabular}

Table 4. Performance of influenza versus COVID-19-positive and influenza versus others (COVID-19-positive/-negative) classification models on the external validation cohort.

\begin{tabular}{lll}
\hline Performance measure & $\begin{array}{l}\text { COVID-19-positive vs } \\
\text { influenza }\end{array}$ & Influenza vs others \\
\hline AUC & 0.928 & 0.914 \\
Accuracy & 0.858 & 0.863 \\
F1 score & 0.838 & 0.874 \\
Sensitivity (recall) & 0.806 & 0.931 \\
Specificity & 0.901 & 0.791 \\
PPV (precision) & 0.872 & 0.824 \\
NPV & 0.847 & 0.916 \\
FPR & 0.099 & 0.209 \\
FDR & 0.128 & 0.176 \\
FNR & 0.194 & 0.069 \\
\hline
\end{tabular}

(54\%), while the influenza group involved more female $(53 \%)$ patients. The COVID-19-positive group included more Black/ African-American (47\%) patients, while the influenza cohort consisted of more White/Caucasian (52\%) patients. The vital signs for mean body temperature, heart rate, respiratory rate, and diastolic blood pressure showed a statistical difference between patients with COVID-19 and those with influenza $(p<0.0001)$.

\section{COVID-19 versus influenza infection prediction at the ED or hospitalization}

In this study we explored the value of demographics, vitals and symptomatic features, which are readily available to providers, in an effort to develop supervised machine learning classifiers that can predict patients who are either COVID-19 positive or negative, while further distinguishing influenza from COVID-19 infection. The WVU patient cohort was randomly divided into a training (80\%) and testing set (20\%) to develop four different context specific XGBoost predictive models. We assessed receiver operator characteristic (ROC) area under the curve (AUC) plots, precision, recall, and other threshold evaluation metrics to select the best performing model in each case (Tables 3 and 4, Supplementary Table 3).

Figure $1 \mathrm{a}, \mathrm{b}$ shows the ROC curves for the prediction of influenza from COVID-19 positive encounters and from those who are either COVID-19 positive or negative obtained using the holdout test set. We present four unique models that can be used as a framework to aid in the delineation between influenza and COVID-19 in the clinical setting. The first model provided stratification of patients as either influenza or COVID-19 positive, highlighted by a ROC AUC $98.8 \%$, accuracy of $95 \%$, sensitivity $93 \%$, and specificity $96 \%$ at identifying COVID-19-positive patients (Table 3). The second model distinguished influenza patients from all other patients, irrespective of a patient's COVID-19 test, revealing an ROC AUC of $98.6 \%$, accuracy of $95 \%$, sensitivity of $91 \%$, and specificity of $96 \%$ (Fig. 1b). The third model distinguishes between COVID-19 positive and negative patients, with a ROC AUC of $97.3 \%$, accuracy of $93 \%$, sensitivity of $87 \%$, and specificity of $95 \%$ (Fig. 2a and Table 3). The Precision-Recall AUC was 93\% for predicting COVID-19-positive versus -negative patients (Supplementary Fig. 3).

The fourth model employed a multi-class XGBoost framework trained to distinguish between all three different types of patients had an ROC AUC of $98 \%$ and achieved $91 \%$ precision at identifying patients that were positive for influenza with 91\% recall, and $94 \%$ precision at identifying those patients that tested negative for COVID-19 with a $88 \%$ recall. While the highest specificity with precision was achieved when identifying COVID-19-positive patients, the recall of $78 \%$ was significantly lower compared to other classes (91\% for influenza and $88 \%$ for COVID-19-negative patients). Average precision of the overall model was $91 \%$, accuracy was $90 \%$, and macro-average of ROC AUC was $97.6 \%$ (Fig. 2 and Supplementary Table 3).

\section{Importance of various features}

We use the SHapley Additive exPlanations (SHAP) method ${ }^{15,16}$, specifically, using the Tree Explainer method, to describe our XGBoost models.

SHAP is a model-agnostic interpretability method that aids in analyzing feature importance based on their impact on the model's output. The additive importance of each feature for the model is calculated over all possible orderings of features. Positive SHAP values indicate a positive impact on the model's output, while negative values indicate negative impact on the model's output. The most important features that identify patients with positive influenza infection from others that are presented to ED included features such as month of encounter, age, body temperature, body surface area (BSA), and heart rate (Fig. 3a, b). Encounter-related features such as encounter type along with reason for visit (Supplementary Table 4) also contributed to the most-informative variables for predicting influenza compared to COVID-19-positive patients or influenza vs all other patients (Fig. 3). On the contrary, in the case of the COVID-19-positive vs COVID-19-negative model, two vital signs i.e., body temperature and $\mathrm{SPO}_{2}$ were amongst the highest-ranking features. BMI, blood pressure, heart rate, respiration rate, and encounter-related variables such as reason for visit and month of encounter were additionally amongst the variables most informative to model predictions. A similar trend of feature importance was also reflected in the SHAP summary plot of the three-way multi-class classifier (Supplementary Fig. 2). Vital signs played a more significant role in distinguishing between influenza and COVID19-positive encounters through parameters such as body temperature, heart rate, and blood pressure.

From the SHAP summary plots (Fig. 3 \& Supplementary Fig. 2), it is evident that the models captured some important features and patterns that aid in correctly predicting influenza and COVID-19positive encounters. These plots highlighted that patient encounters predicted to be COVID-19-positive are, on average, more likely to have lower heart rate, higher respiratory rate, and lower oxygen saturation. Lower blood oxygen saturation is known to be prevalent among COVID-19 patients and was identified by our 
a. Influenza vs COVID-19-pos

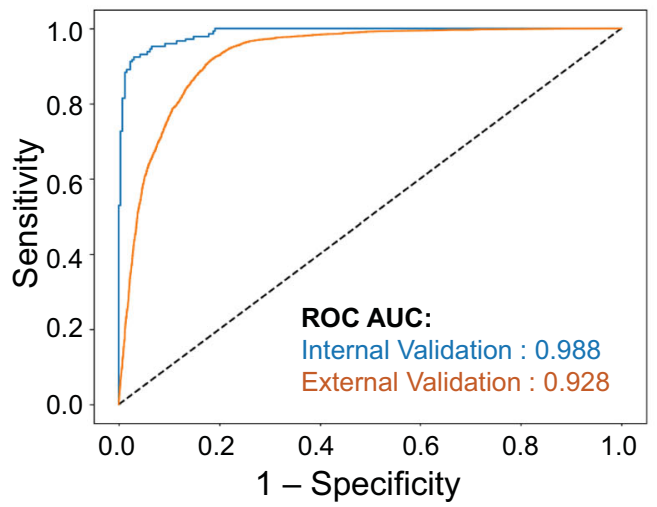

b. Influenza vs Others

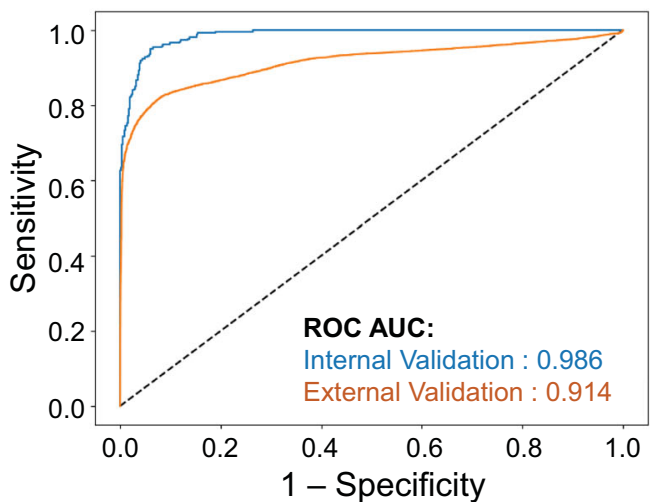

Fig. 1 Receiver operating characteristic (ROC) curves of influenza vs COVID-19-positive and influenza vs other predictive models. Area under the ROC curve showing the predictive performance of the a influenza versus COVID-19-positive model and $\mathbf{b}$ influenza versus other prediction models on the internal test and external validation datasets.
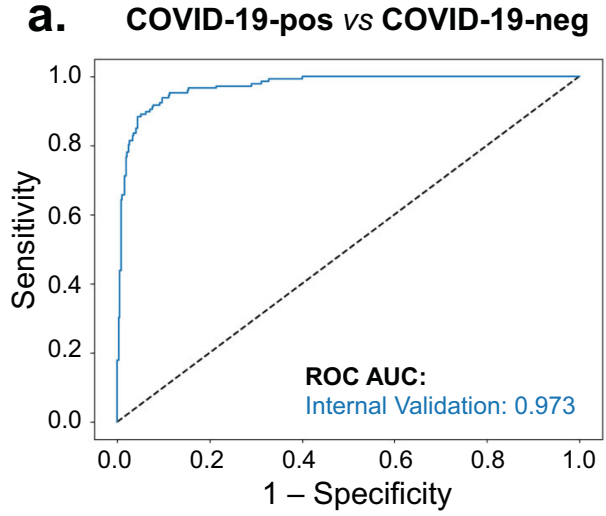

b.

Influenza vs COVID-19-pos vs COVID-19-neg

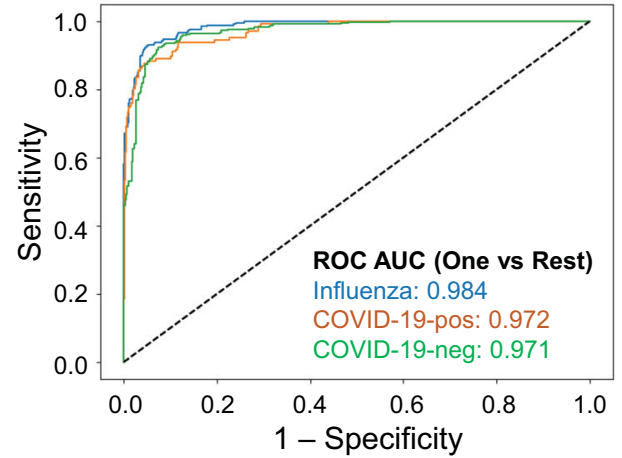

Fig. 2 Receiver operating characteristic (ROC) curves for COVID-19-positive vs COVID-19-negative and multi-class predictive models. Area under the ROC curve showing the predictive performance of the $\mathbf{a}$ COVID-19-positive versus COVID-19-negative, and $\mathbf{b}$ influenza versus other prediction models on the internal validation test.

machine learning models as an important feature for validating the clinical appropriateness of our model design.

The SHAP force plots shown in Figs. 4 and 5 aim to offer explanations for individual predictions made by our models. The two plots generated for the COVID-19-positive vs influenza classifier are shown in Fig. 4. Figure 4a shows an encounter correctly classified as influenza, with the month (during the time course of the traditional influenza season), BSA, and age (young adult patient) values affecting the model output most. The effect of these features was enough to prevent misclassification due to relatively lower heart rate and body temperature values. Figure $4 \mathrm{~b}$ explains an encounter correctly classified as COVID-19. While the encounter occurred in month 3 , which overlaps with the influenza season, the combined effect of higher age, relatively low heart rate, BSA, and higher respiration rate pushes the prediction towards COVID-19, counteracting the effect of month, reason for visit, and encounter type.

Similarly, a second set of plots were generated for the COVID19-positive vs -negative classifier (Fig. 5). In these plots, Fig. 5a shows an encounter correctly classified as COVID-19 negative while Fig. 5b shows an encounter classified correctly as COVID-19 positive. We see in the former that the temperature, reason for visit, lower age, and higher value of $\mathrm{SPO}_{2}$ push the prediction towards COVID-19 negative. In the latter, we see that the effect of temperature, heart rate, and low $\mathrm{SPO}_{2}$ value together counter the effect of the normal respiratory rate, to correctly classify the example as COVID-19 positive. With these interpretability methods we are able to clearly determine the reasons for the model's output and ensure they can be scrutinized. The insights obtained further corroborate with patterns often observed in COVID-19 patients (Fig. 3c).

\section{Impact of vitals on model performance}

As a first step each feature's importance to the construction of the machine learning model was assessed through individually removing each vital sign parameter in the internal validation set. Stepwise removal of vitals in the order of their importance of contribution to the multi-class classifier (Supplementary Fig. 2) led to decrease in the performance of the model (Supplementary Fig. 4a). Removal of body temperature significantly decreased models' performance to an accuracy of $75 \%$ and $\mathrm{F} 1$ score of $70 \%$ compared to the initial performance $90 \%$ and $89 \%$, respectively. However, subsequent removal of other vital signs, including heart rate did not affect model performance drastically.

We also assessed how well the model performs given only one vital is present at a time in the internal test set. More specifically, when considering the importance of a given vital sign, all other features related to vitals are removed from the internal test set. However, the demographics and other information was not 


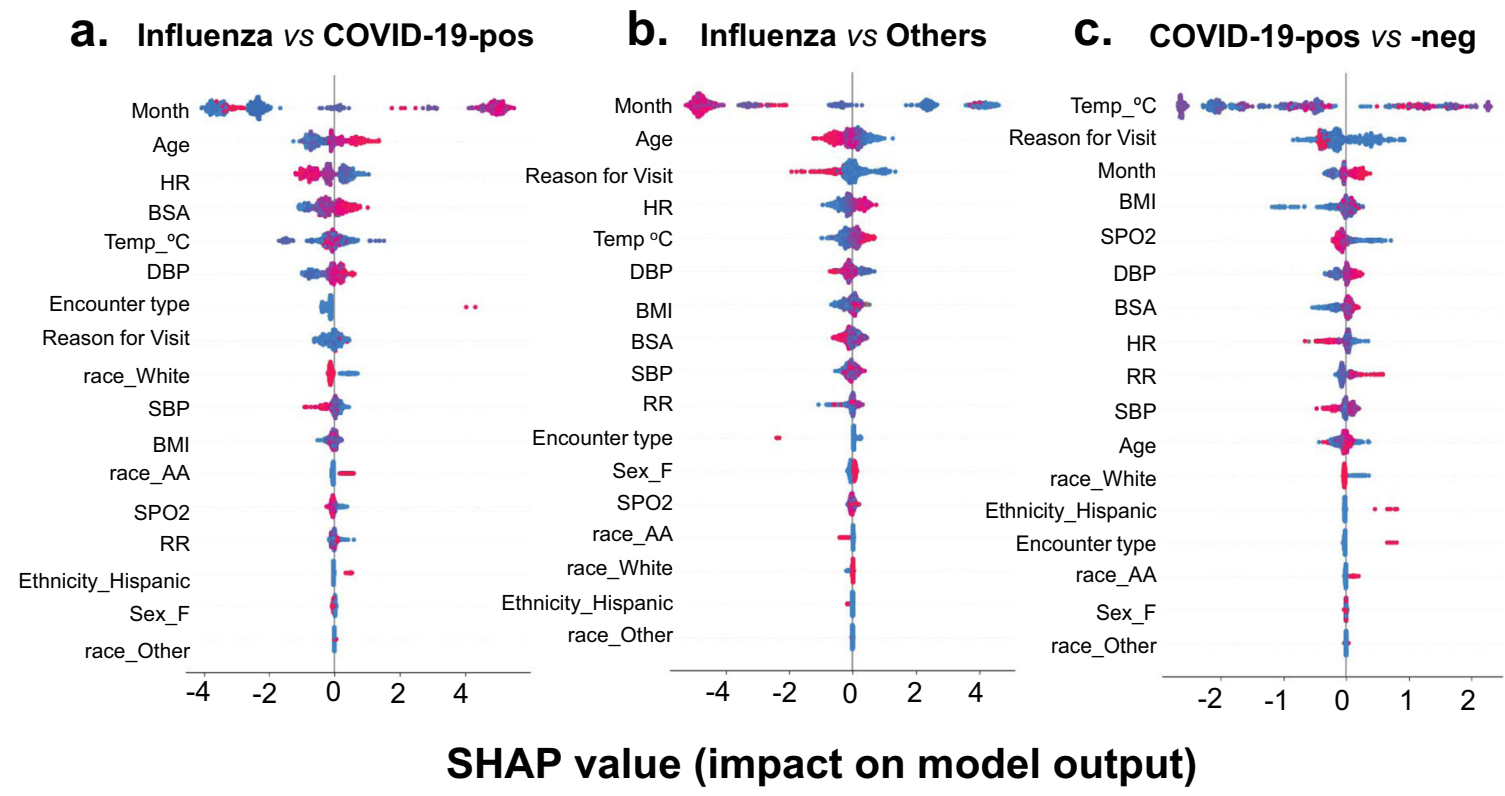

Fig. 3 SHapley Additive exPlanations (SHAP) beeswarm summary plot of SHAP values distribution of each feature of the test dataset. The plot depicts the relative importance, impact, and contribution of different features on the output of a influenza vs COVID-19-positive, b influenza vs other, and c COVID-19-positive vs COVID-19-negative predictive models. The summary plot combines feature importance with feature effects. The features on the $y$-axis are ordered according to their importance. Each point on the summary plot is a SHapley value for a feature and an instance (i.e., a single patient encounter in this case) in the dataset. The position of each point on the $x$-axis shows the impact that feature has on the classification model's prediction for a given instance. The color represents the high (red) to low (blue) values of the feature (i.e., Age, BMI etc.).
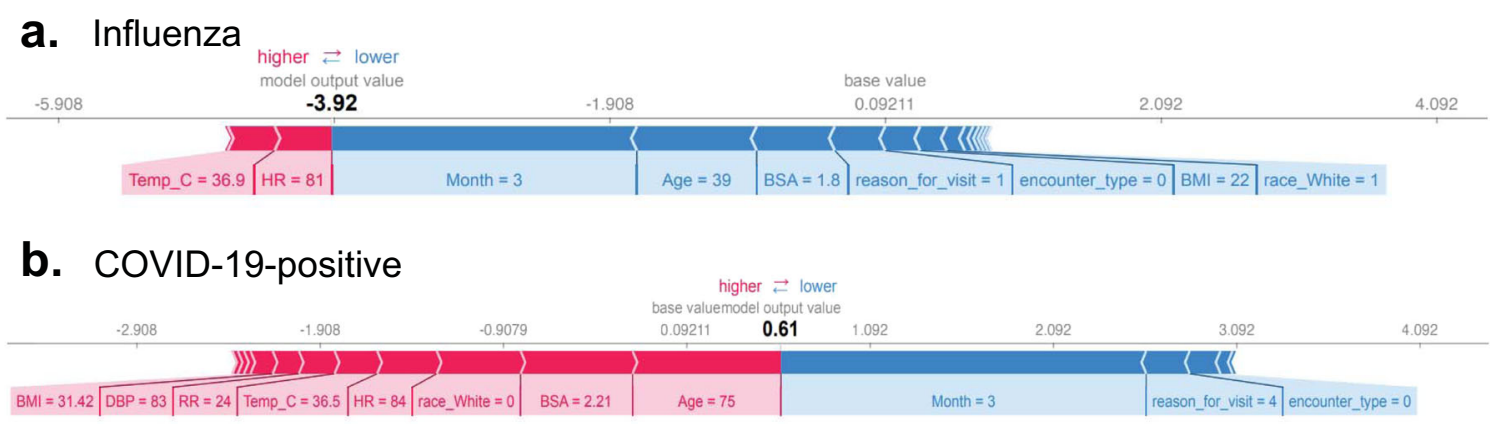

Fig. 4 SHapley Additive exPlanations (SHAP) force plots from an influenza and COVID-19-positive patient. SHAP plots for sample observations from a influenza A/B and b COVID-19-positive predictions. The underlying model is XGBoost. Features that are contributing to a higher and lower SHAP values are shown in red and blue, respectively, along with the size of each feature's contribution to the classification model's output. The influenza patient in this example shows a log-odds output of -3.92 in the rating scale, which is equal to a probability of 0.0194. The baseline-the mean of the model output (log-odds) over the training dataset-is 0.092 (translating to a probability of 0.523 ). The COVID-19-positive patient has a rating score of 0.61 (probability $=0.6479$ ); Age of 75, HR of 84, BSA of 2.21, respiratory rate of 24, and a Temp of $36.5^{\circ} \mathrm{C}$ increases the prediction risk, while month of march decreases the predicted risk of being COVID-19 positive.

ablated. Supplementary Fig. 4b shows the models performance in terms of F1 score or statistic by only including one vital at a time. The higher the performance, the more useful the vital sign is in helping the model to discriminate between COVID-19 and influenza encounters. With the comparison of both multi-class classifier and COVID-19-positive vs -negative classifier performances, it is evident that body temperature has the greatest impact among all vital signs considered. In addition to body temperature, heart rate was also seen to contribute to the performance of COVID-19-positive vs -negative model.

Taken together, these results suggest that of all the vital signs, body temperature, followed by heart rate and $\mathrm{SPO}_{2}$, could impact the predictive models' performance in discriminating between influenza, COVID-19-positive and -negative patient encounters.

\section{External validation}

To further assess the generalizability of the predictive models and confirm the stability of the model features at identifying patients positive for COVID-19 or influenza, we validated our models using the TriNetX research network dataset external to WVU Medicine. Patients with any missing data related to body temperature were excluded from the analysis. The dataset included 6613 encounters of COVID-19 patients $(n=1057)$ and 9084 encounters related to influenza patients $(n=2068)$. The influenza versus COVID-19 model demonstrated ROC AUC of $92.8 \%$ with an accuracy of $86 \%$, and $87 \%$ precision at identifying patients that were positive for COVID-19 with $81 \%$ recall (Fig. $1 \mathrm{a}$ and Table 4). Also, the model to detect patients with influenza, irrespective of their COVID-19 status, showed similar performance with an AUC ROC of $91.4 \%$ 


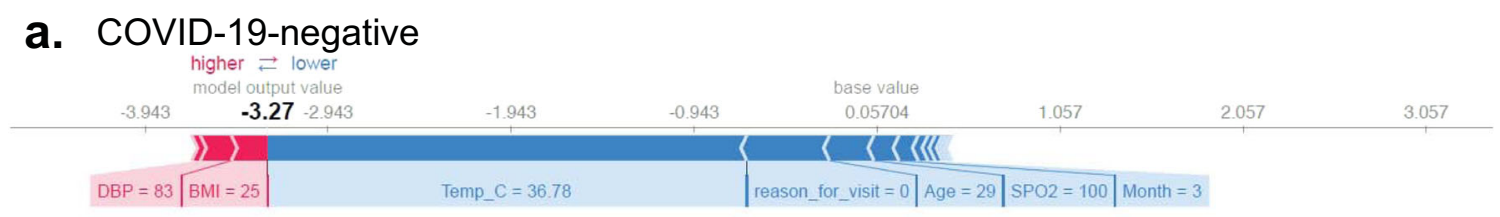

\section{b. COVID-19-positive}

Fig. 5 SHapley Additive exPlanations (SHAP) force or explanation plots of COVID-19-positive and -negative patient encounters. Sample observations from two patient encounters with a COVID-19-negative and $\mathbf{b}$ COVID-19-positive predictions. Features that are contributing to a higher and lower SHAP values are shown in red and blue, respectively, along with the size of each feature's contribution to the model's output. The baseline-the mean of the model output (log-odds) over the training dataset-is 0.057 (translating to a probability value of 0.5143 ). The first patient-who is COVID-19 negative-has a low predicted risk score of -3.27 (output probability $=0.0366$ ). The second patient-who is COVID-19 positive-has a high predicted risk of 1.94 (output probability $=0.8744$ ). These SHAP output values represent a "raw" log-odds value which is transformed into a probability space, to provide the final output of 0 and $1(<0.5$ and $>0.5)$. Risk increasing effects such as Temp, $\mathrm{SpO}_{2}, \mathrm{HR}$, and BP were offset by decreasing effects of RR in pushing the model's predictions towards or away from the positive class, respectively.

and an accuracy of $86 \%$, and $82 \%$ precision at identifying patients that were positive for influenza with $93 \%$ recall (Fig. 1b). This suggests that the developed models are able to effectively identify patients across multiple TriNetX HCOs with influenza or COVID-19 infections amongst other COVID-19-negative patients presenting at $\mathrm{ED}$ and/or admitted to the hospital.

Further, enforcing no missing values in the case of both heart rate and body temperature, the two top ranked vital signs (Supplementary Fig. 4), did not result in boosting the performance. On the contrary, restricting the data to have all vitals present, boosted the performance of ROC AUC to $94.9 \%$ and F1score to $87 \%$ for the influenza vs COVID-19 model and ROC AUC to $95.4 \%$ and $\mathrm{F} 1$-score to $89 \%$ in the case of influenza vs other model (Supplementary Table 5). These results suggest that while enforcing no missing values in vitals could support a better overall model performance (i.e., AUC ROC 94.9\% vs 92.8\%), missingness in most of the vitals does not seem to limit its applicability and generalizability.

\section{DISCUSSION}

This investigation provides multiple machine-learning models to differentiate between COVID-19-positive, -negative, and influenza. Further, this is the first machine learning model to leverage a patient population that includes both the initial (February-April) and secondary (May-September) surge of SARS-CoV 2 infections in the United States ${ }^{17}$. Our approach can guide future applications, highlighting the importance of developing dynamic models that control for confounding comorbidities, such as influenza, and the ever-evolving infectivity of SARS-CoV 2. Importantly, as influenza season approaches it will be of high priority to establish a reliable process for identifying patients at risk of COVID-19, influenza, or other viral infections to increase the prognostic value of a directed therapy.

While the initial presentation of COVID-19 and influenza appear similar, the number, and combination, of signs and symptoms can help provide better stratification. From a symptom standpoint, COVID-19 causes more fatigue, diarrhea, anosmia, acute kidney injury, and pulmonary embolisms, while sputum production and nasal congestion are more specific to influenza ${ }^{18}$. Furthermore, COVID-19 tends to cause worse decompensation. For instance, out of 200 cases of either influenza or COVID-19 in a study from France, only COVID-19 resulted in severe respiratory failure requiring intubation ${ }^{18}$. Our evaluation revealed that the month the patient was seen, age, and heart rate were the most important features for predicting a diagnosis of COVID-19 over that of influenza infection. Additionally, body temperature and $\mathrm{SPO}_{2}$ were two of the most important features that indicated if a patient would be positive or negative for COVID-19, which is likely a result of the underlying pathophysiology of the virus. SARS-CoV 2 infection appears to cause more significant endothelial dysfunction and systemic inflammation likely leading to the worse casefatality rate ${ }^{6,19}$. These differences can be seen histologically as well. Autopsy samples of COVID-19 lung tissue showed severe endothelial injury and widespread thrombosis with microangiopathy ${ }^{20}$. These samples had nine times as much alveolar capillary microthrombi compared to that of influenza lung tissue and even showed evidence of new vessel growth through a mechanism of intussusceptive angiogenesis ${ }^{1,20}$.

Machine learning algorithms can provide distinct advantages in the classification of positive COVID-19 cases as the virus, and the demographic it infects, continues to evolve. The West Virginia University (WVU) cohort consists of a fairly homogenous population, with $89 \%$ of the population identifying as White/Caucasian and only $5 \%$ as Black/African American. While our machine learning models were built around the WVU cohort, they demonstrate robust performance even on more racially/ethnically diverse populations, such as in the TriNetX dataset, when predicting patients who have influenza or COVID-19 (ROC AUC $=92.8 \%$ ) and influenza only (ROC AUC $=91.4 \%$ ). The generalizability of our model may suggest that features that define SARSCoV 2 infection (e.g., age, month of encounter, heart rate, body temperature, $\mathrm{SPO}_{2}$ ) could represent similarities in clinical presentation, regardless of a patient's race/ethnicity. Further understanding the socioeconomic ${ }^{21,22}$ and physiological ${ }^{23,24}$ differences in minority populations will be important in determining why COVID-19 disproportionately affects these populations and how machine learning models can more accurately model heterogeneous populations.

Patient information provided in the WVU and TriNetX datasets consists of both the initial rise in cases in the United States (February-April) as well as a secondary surge (May-September). The second surge of infections shifted demographically from a predominately older population to one with the highest prevalence between ages 20-29, with an increased rate of mild to asymptomatic presentations ${ }^{25}$. Additionally, SARS-CoV 2 is an RNA virus with the capacity to mutate. Sequencing studies have already identified a variety of genetic variants, with most mutations currently having no clear association with a positive or negative selective virulence ${ }^{26}$. However, some mutations, such 
as D614G, are suggested to increase infectivity of SARS-CoV 2 and outcompete other strains in the environment ${ }^{27}$. Regardless, in our current approach, applying machine learning over the continuum of the COVID-19 epidemic provides distinct advantages in producing a generalizable model for continued use, which may not be captured in models generated on earlier datasets.

The current study provides robust ROC AUC prediction of patients with COVID-19 (97.2\%), without COVID-19 (97.1\%), and those with influenza (98.4\%) in our multi-class model (Fig. 2b). While only demographic information and vital signs along with encounter-related features were applied to our models, overall prediction could likely improve with the addition of other features including biochemical, metabolic, and molecular markers, as well as imaging modalities such as X-ray and CT. The addition of these features could likely improve diagnostic accuracy and should be tested in future studies delineating between COVID-19 and influenza. Additionally, the current investigation involves influenza and COVID-19 data from non-overlapping periods (i.e., influenza from winter 2019 and COVID-19 from spring/summer/fall 2020). Although some of the machine learning models rely on time of presentation for prediction, we anticipate that the four-model framework provided in the study will sufficiently stratify patients even when influenza season and the COVID-19 epidemic overlap. This is reinforced by the prediction of COVID-19-positive and -negative cases (ROC AUC $=97.3 \%$ ) in our model, which did not rely on time of presentation as a variable. In addition, similar performance in AUC-ROC curves was observed when the different models were either evaluated on patient groups separated by their encounters occurring in different seasonal months (Table 3 and Supplementary Fig. 5) or retrained on season instead of month of encounter as a feature (Supplementary Table 6).

Our study was limited by the classification of patients in the internal (WVU) and external (TriNetX) datasets. While the WVU cohort includes patients, who have been confirmed to have a negative SARS-CoV 2 test on presentation, the TriNetX dataset does not have this information. Both datasets are also unable to capture patients with other respiratory viruses and viral coinfections; information that could better explain variations in COVID-19 clinical presentation. Another limitation is the need to continually adjust these models to fit new trends in SARS-CoV 2 spread and infectivity. Our models benefit from the inclusion of data spanning from February to October, which can better simulate the current COVID-19 epidemic and influenza season but will still require future iterations and validations to accurately predict SARS-CoV 2 infections. Removal or ablation of month of encounter during the validation step resulted in a significant drop in AUC (decreased from $98.8 \%$ to $84 \%$ ), suggesting the interactions between months and vital signs is important for differentiating influenza patients from COVID-19. Seasonality can induce variations in vital signs including body temperature, blood pressure ${ }^{28}$, and in turn these can affect and influence the different disease populations (e.g., diabetes, cardiovascular, obesity, and others) ${ }^{29-31}$. A seasonal variation of deaths from corona viruses was also observed recently ${ }^{32}$. Thus, the months/seasons may have important interactions with the vital signs beyond the known seasonal susceptibility to influenza. Nevertheless, we observed robust performance of the model if the analysis was restricted for differentiating COVID-19 vs influenza for the same month (e.g., January) or if seasons were included in place of specific month related information (Supplementary Table 6). Future studies focusing on such details and sub-group analysis is warranted to confirm the relevance of different model predictions. All the models will be made available at https://github.com/ynaveena/ COVID-19-vs-Influenza for further assessment by the community since it remains unclear whether COVID-19 may also start showing seasonal trends in the future. Further, an understanding of COVID19 and viral co-infections is needed to appropriately model the risks of patients presenting with both illnesses ${ }^{33-35}$.
Here we highlight how machine learning can effectively classify influenza and COVID-19-positive cases through vital signs on clinical presentation. This work is the first step in building a lowcost, robust classification system for the appropriate triage of patients displaying symptoms of a viral respiratory infection. With these algorithms, the identification of proper treatment modalities for both COVID-19 and influenza can be made more rapidly, increasing the effectiveness of patient care. We certainly hope that our current work can aid healthcare workers and clinicians to rapidly identify, triage, and guide treatment decisions when the two viral infections start cocirculating in the communities.

\section{METHODS}

\section{WVU internal cohort dataset}

This study protocol was reviewed by the West Virginia University Institutional Review Board (WVU IRB) and ethical approval was given. A waiver of consent was granted by the IRB as this is retrospective study. Deidentified clinical and demographics data for all patients presenting to the emergency department and/or admitted at West Virginia University hospitals between January $1^{\text {st }}, 2019$, and November $4^{\text {th }}, 2020$, were extracted and provided to us by WVU Clinical and Translational Science Institute (CTSI). The COVID-19-positive dataset includes all patients who had a lab-based diagnostic test positive for SARS-CoV-2 at WVU Medicine between March $1^{\text {st }}, 2020$, and November $4^{\text {th }}, 2020$ and had an associated hospital encounter with all vital signs information available. The influenzapositive dataset includes a random sample of 1500 patients who had a labbased diagnostic test that was positive for either influenza type $A$ or influenza type B at WVU Medicine between January 1st, 2019, and November 3rd, 2020, and had a hospital encounter associated with that positive test (Supplementary Fig. 1). The COVID-19-negative dataset includes a random sample of 2000 patients who had negative test results for SARS-CoV-2 and who never had a positive diagnostic test for SARS-CoV2 at WVU Medicine between March $1^{\text {st }}, 2020$, and November $4^{\text {th }}, 2020$ provided that an associated hospital encounter occurred with that negative test. As our study aims to create a model that can accurately discriminate COVID-19 from influenza patients solely based on the patient's demographic information and vital signs data, patients with any of the vital signs missing during the associated encounters were therefore excluded from the analysis. This resulted in a final WVU cohort that included a total of 747 COVID-19-positive, 1223 influenza, and 1913 COVID-19-negative patients (Table 1, Supplementary Fig. 1).

\section{TriNetX external cohort dataset}

The external validation cohort was obtained from the TriNetX Research network, a cloud-based database resource that provides researchers access to de-identified patient data from networks of healthcare organizations (HCO), mainly from large academic centers and other data providers. The data is directly pulled from electronic medical records and accessible in a de-identified manner. The TriNetX database includes patient-level demographic, vital signs, diagnoses, procedures, medications, laboratory, and genomic data. For this study, we identified COVID-19 and influenza cases using the International Classification of Diseases, $10^{\text {th }}$ revision. We used ICD-10 codes J9.0, J10.0, J09.*, and J10.* for influenza, and U07.1 for COVID19. Cases from February $1^{\text {st }}, 2020$, to August $13^{\text {th }}, 2020$, were queried and extracted. Due to heterogeneity in reporting of COVID-19 testing (i.e., positive vs negative; normal vs abnormal), we refrained from selecting the patients based on their testing results and used diagnostic codes instead. Thus, we were only able to extract COVID-19-positive and influenzapositive patients for external validation. Further patients at least 18 years or older at the time of diagnosis and their encounters defined as an encounter occurring within 7days from the date of diagnosis were enforced to be included in the final cohort $(n=34,670)$. Enforcing no missing values corresponding to body temperature resulted in a dataset ( $n$ $=15,697)$ that had a total of $6613(n=1057$ patients $)$ and $9084(n=2068$ patients) encounters diagnosed with COVID-19 and influenza, respectively (Supplementary Fig. 1). Baseline characteristics of the cohort is described in Supplementary Table 2. Since the TriNetX data is de-identified, Institutional Board Review (IRB) oversight is not necessary. 


\section{Data preprocessing and split}

Different clinical variables investigated in this study include (a) vital signs i.e., body temperature, heart rate, breathing/respiratory rate, oxygen saturation or $\mathrm{SPO}_{2}$, and blood pressure, (b) encounter date/type, (c) reason for visit, and (d) basic demographics information such as age, gender, race, and ethnicity. Of the features considered, the data elements corresponding to sex and ethnicity were binarized for being "female" and "Hispanic or Latino", respectively. The race feature was further one-hot encoded to three variables i.e., race_White/Caucasian, race_Black/African American, and race_Other. Finally, the date of the encounter was converted to represent a numerical value for month, rather than specific day/year to remove the granularity and capture the seasonal effects of influenza.

\section{Predictive model development using extreme gradient boosting trees}

XGBoost, a gradient-boosted tree algorithm, was used to develop classifiers to distinguish between patient encounters with confirmed COVID-19 from that of either influenza patient encounters or other unknown viral infections. Separate models were developed to predict confirmed COVID-19 patient encounters post Feb 1, 2020 from either influenza and/or those that tested negative for COVID-19 (i.e., COVID-19positive vs influenza, COVID-19-positive vs COVID-19-negative, COVID-19positive vs influenza vs COVID-19-negative), and then an additional model to test influenza infections from others i.e., patients that were either positive or negative to a COVID-19 test (influenza vs others). For the purpose of model training and testing, we only consider data from $01-$ Feb2020 until 04-Nov-2020 for COVID-19-positive and -negative cohorts and from 01-Jan-2019 to 04-Nov-2020 for the influenza patient cohort. We partitioned the dataset into training and testing sets, using an $80 \%-20 \%$ split (Supplementary Table 1). The holdout test, or internal validation, set is never seen by the model during training and was used only during performance evaluation.

Gradient-boosted trees were selected due to their ability to model complex nonlinear relationships, while robustly handling outliers and missing values. Gradient-boosted trees fuse the concepts of gradient descent (in the loss function space) and boosting. Simpler tree-based models are built additively like a boosting ensemble, to fit the gradient of the loss function for every data point. Only a fraction of the trees fit to the gradient of the loss per data point in the training set. This is analogous to small steps of gradient descent in the loss function space.

For developing the COVID-19-positive vs -negative, influenza vs others and COVID-19 vs influenza XGBoost models, the value of learning rate was set to 0.02 and the total number of trees was 600 . The alpha value was set to 0 and lambda was set to 1 , i.e., we use L2 regularization. Further, min_child_weight was set to 1, to allow highly specific patterns to be learnt as well. To counter the possibility of overfitting, the max_depth parameter of the XGBoost model was set to 4 , as more complex features are learnt with higher depths, leading to poor performance on the unseen data. We used the gain importance metric to decide node splits for the tree estimator, and the objective was set to binary logistic regression. The model outputs the predicted probability, and records whose probability was at least 0.5 were considered as belonging to the positive class, while others were deemed to belong to the negative class. Further, the subsample parameter of the XGBoost algorithm represents the ratio of the records to be sampled before forming a tree and was set to a value of 0.8 . Similarly, the colsample_bytree parameter which determines the fraction of columns to be sampled before every tree formation was set to 1 , meaning all features are used. Only records with all vitals were considered; however, the model does have the ability to make predictions in the absence of certain features. The compute_sample_weight from the sklearn.utils.class_weight library was employed to ensure that each training sample is weighted based on its class, to counter imbalance in the dataset.

For the three-class classifier that discriminates between COVID-19positive, COVID-19-negative, and Influenza encounters, we developed an XGBoost model of 100 trees, with learning_rate set to 0.3 and max_depth fixed at 6 . The objective was set to softprob (uses a softmax objective function), to perform multi-class classification. Furthermore, the subsample parameter was set to 1 , meaning all samples were used to form trees. No sample weighting was carried out for this model. All other parameters remained the same as the models discussed previously.

We also consider an unseen external validation dataset (TriNetX cohort) to evaluate the performance of the model on data that is completely different from what it is accustomed to during training. Patient-wise encounters with no missing vitals were enforced to test and evaluate the performance of the developed models.

\section{Model interpretability using SHAP}

To identify the principal features driving the model prediction, SHAP values were calculated. The SHAP method is suitable for the interpretation of complex models such as artificial neural networks and gradient-boosting machines (e.g., XGBoost) ${ }^{36,37}$. Originating in game theory, SHAP provides model output explanations to answer how does a given prediction change when a particular feature is removed from the model. The resulting SHAP values quantify the magnitude and direction (positive or negative) of a feature's effect on a given prediction. Thus, SHAP partitions the prediction result of every sample into the contribution of each constituent feature value by estimating differences between model outputs with subsets of the feature space. By averaging across samples, this method helps estimate the contribution of each feature to overall model predictions for the entire dataset. The SHAP is also representative of how important a feature is to the prediction-larger the absolute SHAP value of the feature, the greater its impact on predictions. The direction of SHAP values in force plots represents whether the feature is influential or indicative for the negative or positive class.

\section{Performance evaluation metrics}

The discriminative ability of each of the models developed in predicting patients with influenza from COVID-19-positive and /or -negative patients as well as those with a positive COVID-19 test from negative test was evaluated in the hold-out test set and an external validation test by using receiver operator characteristic (ROC) curve analysis. An area under the curve $(A \cup C)>0.5$ indicated better predictive values. The closer the AUC was to 1 , the better the model's performance was. Additionally, "classification accuracy", the total number of true positive scores i.e., when predicted values are equal to the actual values given by the attribute positive predictive value (PPV) or "precision" score, "recall" which is the total number of true positive instances among all the positive instances, and " $F 1$ score" the weighted harmonic mean of precision and recall along with negative predictive value (NPV) were estimated to evaluate performance and the overall generalizability of each of the models. Further details on the calculations of different metrics employed are provided in Supplementary methods.

\section{Statistical analysis}

We performed the Shapiro-Wilk test on the entire WVU internal cohort dataset to check for normalcy of the data. We found that all the variables were normally distributed; therefore, we used parametric methods for further statistical analysis. Categorical data are presented as counts (percentages) while continuous data were reported as mean \pm standard deviation (SD). To determine the significance of continuous variables between all three different groups (i.e., COVID-19 positive, influenza, and COVID-19 negative) Kruskal-Wallis test with Dunn-Bonferroni correction test was used. The comparison between two of these three groups (COVID19 positive vs negative, COVID-19 positive vs influenza, influenza vs COVID19 negative) to determine significance was performed using an independent sample $t$-test. Chi-square was used for the categorical variable because the expected value for each cell is greater than 5 . All statistical analysis was performed using Medcalc for Windows, version 19.5.3 (MedCalc Software, Ostend, Belgium), and python for windows, version 3.8.3. A statistical significance level of $p<0.05$ was used for all the tests performed.

\section{Reporting summary}

Further information on research design is available in the Nature Research Reporting Summary linked to this article.

\section{DATA AVAILABILITY}

The data that support the findings of this study are available from WVU CTSI \& TriNetX but restrictions apply to the availability of these data, which were used under license for the current study, and so are not publicly available. Data is however available from the authors upon reasonable request and with permission of WVU CTSI \& TriNetX. 


\section{CODE AVAILABILITY}

The code is made available at https://github.com/ynaveena/COVID-19-vs-Influenza for non-commercial use.

Received: 20 November 2020; Accepted: 6 May 2021;

Published online: 04 June 2021

\section{REFERENCES}

1. Ackermann, M. et al. Pulmonary vascular endothelialitis, thrombosis, and angiogenesis in Covid-19. N. Engl. J. Med. 383, 120-128 (2020).

2. Li, Q. et al. Early transmission dynamics in Wuhan, China, of novel coronavirusinfected pneumonia. N. Engl. J. Med. 382, 1199-1207 (2020).

3. Zhu, N. et al. A novel coronavirus from patients with pneumonia in China, 2019. N. Engl. J. Med. 382, 727-733 (2020)

4. Lee, Y. H., Hong, C. M., Kim, D. H., Lee, T. H. \& Lee, J. Clinical course of asymptomatic and mildly symptomatic patients with Coronavirus disease admitted to community treatment centers, South Korea. Emerg. Infect. Dis. 26, 2346-2352 (2020).

5. Gostin, L. O. \& Salmon, D. A. The dual epidemics of COVID-19 and influenza: vaccine acceptance, coverage, and mandates. JAMA 324, 335-336 (2020).

6. Solomon, D. A., Sherman, A. C. \& Kanjilal, S. Influenza in the COVID-19 Era. JAMA https://doi.org/10.1001/jama.2020.14661 (2020).

7. Zayet, S. et al. Clinical features of COVID-19 and influenza: a comparative study on Nord Franche-Comte cluster. Microbes Infect. https://doi.org/10.1016/j. micinf.2020.05.016 (2020).

8. Lalmuanawma, S., Hussain, J. \& Chhakchhuak, L. Applications of machine learning and artificial intelligence for Covid-19 (SARS-CoV-2) pandemic: a review. Chaos Solitons Fractals 139, 110059 (2020).

9. Soltan, A. A. S. et al. Artificial intelligence driven assessment of routinely collected healthcare data is an effective screening test for COVID-19 in patients presenting to hospital. Lancet https://doi.org/10.1101/2020.07.07.20148361 (2020).

10. Sun, L. et al. Combination of four clinical indicators predicts the severe/critical symptom of patients infected COVID-19. J. Clin. Virol. 128, 104431 (2020).

11. Wu, J. et al. Rapid and accurate identification of COVID-19 infection through machine learning based on clinical available blood test results. Preprint at bioRxiv https://doi.org/10.1101/2020.04.02.20051136; (2020).

12. Ardakani, A. A., Kanafi, A. R., Acharya, U. R., Khadem, N. \& Mohammadi, A. Application of deep learning technique to manage COVID-19 in routine clinical practice using CT images: results of 10 convolutional neural networks. Comput. Biol. Med. 121, 103795 (2020).

13. Ozturk, T. et al. Automated detection of COVID-19 cases using deep neural networks with X-ray images. Comput. Biol. Med. 121, 103792 (2020).

14. Diehl, J. L. et al. Respiratory mechanics and gas exchanges in the early course of COVID-19 ARDS: a hypothesis-generating study. Ann. Intensiv. Care 10, 95 (2020).

15. Lundberg, S. M., Lee Su-In in 31st Conference on Neural Information Processing Systems (NIPS 2017), Long Beach, CA, USA.

16. Lundberg, S. M. et al. From local explanations to global understanding with explainable Al for trees. Nat. Mach. Intell. 2, 56-67 (2020).

17. Roser, M., Ritchie, H., Ortiz-Ospina, E. \& Hasell, J. (Published online at OurWorldlnData.org, 2020).

18. Faury, $H$. et al. Medical features of COVID-19 and influenza infection: a comparative study in Paris, France. J. Infect. https://doi.org/10.1016/j.jinf.2020.08.017 (2020).

19. Vardeny, O., Madjid, M. \& Solomon, S. D. Applying the lessons of influenza to COVID-19 during a time of uncertainty. Circulation 141, 1667-1669 (2020).

20. Calabrese, F. et al. Pulmonary pathology and COVID-19: lessons from autopsy. The experience of European pulmonary pathologists. Virchows Arch. 477, 359-372 (2020).

21. Chowkwanyun, M. \& Reed, A. L. Jr. Racial health disparities and Covid-19-caution and context. N. Engl. J. Med. 383, 201-203 (2020).

22. Selden, T. M. \& Berdahl, T. A. COVID-19 and racial/ethnic disparities in health risk, employment, and household composition. Health Aff. 39, 1624-1632 (2020).

23. Fei, K. et al. Racial and ethnic subgroup disparities in hypertension prevalence, New York City health and nutrition examination survey, 2013-2014. Prev. Chronic Dis. 14, E33 (2017)

24. Forde, A. T. et al. Discrimination and hypertension risk among African Americans in the Jackson heart study. Hypertension 76, 715-723 (2020).

25. Boehmer, T. K. et al. Changing age distribution of the COVID-19 pandemic-United States, May-August 2020. Morb. Mortal. Wkly Rep. 69, 1404-1409 (2020).
26. Day, T., Gandon, S., Lion, S. \& Otto, S. P. On the evolutionary epidemiology of SARS-CoV-2. Curr. Biol. 30, R849-R857 (2020).

27. Zhang, L. et al. SARS-CoV-2 spike-protein D614G mutation increases virion spike density and infectivity. Nat. Commun. 11, 6013, https://doi.org/10.1038/s41467020-19808-4 (2020).

28. Stergiou, G. S. et al. Seasonal variation in blood pressure: evidence, consensus and recommendations for clinical practice. Consensus statement by the European Society of Hypertension Working Group on blood pressure monitoring and cardiovascular variability. J. Hypertens. 38, 1235-1243 (2020).

29. Argha, A., Savkin, A., Liaw, S. T. \& Celler, B. G. Effect of seasonal variation on clinical outcome in patients with chronic conditions: analysis of the Commonwealth Scientific and Industrial Research Organization (CSIRO) national telehealth trial. JMIR Med. Inform. 6, e16 (2018).

30. So, J. Y. et al. Seasonal and regional variations in chronic obstructive pulmonary disease exacerbation rates in adults without cardiovascular risk factors. Ann. Am Thorac. Soc. 15, 1296-1303 (2018).

31. Stewart, S., Keates, A. K., Redfern, A. \& McMurray, J. J. V. Seasonal variations in cardiovascular disease. Nat. Rev. Cardiol. 14, 654-664 (2017).

32. Park, S., Lee, Y., Michelow, I. C. \& Choe, Y. J. Global seasonality of human coronaviruses: a systematic review. Open Forum Infect. Dis. 7, ofaa443 (2020).

33. Ding, Q., Lu, P., Fan, Y., Xia, Y. \& Liu, M. The clinical characteristics of pneumonia patients coinfected with 2019 novel coronavirus and influenza virus in Wuhan China. J. Med. Virol. https://doi.org/10.1002/jmv.25781 (2020).

34. Miatech, J. L., Tarte, N. N., Katragadda, S., Polman, J. \& Robichaux, S. B. A case series of coinfection with SARS-CoV-2 and influenza virus in Louisiana. Respir. Med. Case Rep. 31, 101214 (2020).

35. Zhou, X. et al. The outbreak of Coronavirus disease 2019 interfered with influenza in Wuhan. SSRN https://doi.org/10.2139/ssrn.3555239 (2020).

36. Lundberg, S. M. et al. Explainable machine-learning predictions for the prevention of hypoxaemia during surgery. Nat. Biomed. Eng. 2, 749-760 (2018).

37. Lundberg, S. \& Lee, S.-I. A unified approach to interpreting model predictions. Preprint at https://arXiv.org/quant-ph/1705.07874 [cs.Al] (2017)

\section{ACKNOWLEDGEMENTS}

The authors acknowledge Matthew Armistead, Emily Morgan, Maryam Khodaverdi, and Wes Kimble for their support with data collection and providing de-identified datasets. This work is supported in part by funds from the National Science Foundation (NSF: \# 1920920) and National Institute of General Medical Sciences of the National Institutes of Health under (NIH: \#5U54GM104942-04). The content is solely the responsibility of the authors and does not necessarily represent the official views of the National Institutes of Health or National Science Foundation.

\section{AUTHOR CONTRIBUTIONS}

P.P.S. and N.Y. envisioned and designed the projects. N.Y., N.H.K., A.R., and S.S. performed machine learning modeling and analysis. P.P.S., Q.A.H., B.P., and P.F. were involved with clinical interpretation of the findings and writing as well as editing the manuscript. H.B.P. and S.S. performed the statistical analysis. All the authors have significantly been involved in the drafting of the manuscript or revising it critically for important intellectual content.

\section{COMPETING INTERESTS}

The authors declare the following competing interests. Dr. Sengupta is a consultant for Kencor Health and Ultromics. The remaining authors declare no competing interests.

\section{ADDITIONAL INFORMATION}

Supplementary information The online version contains supplementary material available at https://doi.org/10.1038/s41746-021-00467-8.

Correspondence and requests for materials should be addressed to N.Y. or P.P.S.

Reprints and permission information is available at http://www.nature.com/ reprints

Publisher's note Springer Nature remains neutral with regard to jurisdictional claims in published maps and institutional affiliations. 
(c) Open Access This article is licensed under a Creative Commons Attribution 4.0 International License, which permits use, sharing, adaptation, distribution and reproduction in any medium or format, as long as you give appropriate credit to the original author(s) and the source, provide a link to the Creative Commons license, and indicate if changes were made. The images or other third party material in this article are included in the article's Creative Commons license, unless indicated otherwise in a credit line to the material. If material is not included in the article's Creative Commons license and your intended use is not permitted by statutory regulation or exceeds the permitted use, you will need to obtain permission directly from the copyright holder. To view a copy of this license, visit http://creativecommons. org/licenses/by/4.0/.

(c) The Author(s) 2021 